\title{
Association of the fat mass and obesity-associated (FTO) gene variant (rs9939609) with dietary intake in the Finnish Diabetes Prevention Study
}

\author{
Tiina Lappalainen $^{1 *}$, Jaana Lindström ${ }^{2,3}$, Jussi Paananen ${ }^{1}$, Johan G. Eriksson ${ }^{2,4,5,6,7}$, Leila Karhunen ${ }^{1}$, \\ Jaakko Tuomilehto $^{2,3,8}$, Matti Uusitupa ${ }^{1,9}$ for the Finnish Diabetes Prevention Study Group \\ ${ }^{1}$ Department of Clinical Nutrition, Institute of Public Health and Clinical Nutrition, University of Eastern Finland, \\ Kuopio Campus, PO Box 1627, FI-70211 Kuopio, Finland \\ ${ }^{2}$ Department of Health Promotion and Chronic Disease Prevention, National Institute for Health and Welfare, Helsinki, \\ Finland \\ ${ }^{3}$ Department of Public Health, University of Helsinki, Helsinki, Finland \\ ${ }^{4}$ Department of General Practice and Primary Health Care, University of Helsinki, Helsinki, Finland \\ ${ }^{5}$ Unit of General Practice, Helsinki University Central Hospital, Helsinki, Finland \\ ${ }^{6}$ Folkhalsan Research Centre, Helsinki, Finland \\ ${ }^{7}$ Vasa Central Hospital, Vasa, Finland \\ ${ }^{8}$ South Ostrobothnia Central Hospital, Seinäjoki, Finland \\ ${ }^{9}$ Research Unit, Kuopio University Hospital, Kuopio, Finland
}

(Submitted 11 August 2011 - Final revision received 25 November 2011 - Accepted 13 December 2011 - First published online 23 January 2012)

\section{Abstract}

A cluster of variants in the fat mass and obesity-associated (FTO) gene are associated with the common form of obesity. Well-documented dietary data are required for identifying how the genetic risk can be modified by dietary factors. The objective of the present study was to investigate the associations between the FTO risk allele (rs9939609) and dietary intake, and to evaluate how dietary intake affects the association between FTO and BMI in the Finnish Diabetes Prevention Study during a mean follow-up of 3.2 years. A total of 479 (BMI $>25 \mathrm{~kg} / \mathrm{m}^{2}$ ) men and women were genotyped for rs9939609. The participants completed a $3 \mathrm{~d}$ food record at baseline and before every annual study visit. The average intakes at baseline and during the years 1, 2 and 3 were calculated. At baseline, the FTO variant rs9939609 was not associated with the mean values of total energy intake, macronutrients or fibre. At baseline, a higher BMI by the FTO risk genotype was detected especially in those who reported a diet high in fat with mean BMI of 30.6 (SD 4.1), 31.3 (SD 4.6) and $34.5(\mathrm{sD} 6.2) \mathrm{kg} / \mathrm{m}^{2}$ for TT, TA and AA carriers, respectively $(P=0.005)$. Higher BMI was also observed in those who had a diet low in carbohydrates $(P=0.028)$ and fibre $(P=0.015)$. However, in the analyses adjusted for total energy intake, age and sex, significant interactions between FTO and dietary intakes were not found. These findings suggest that the association between the FTO genotype and obesity is influenced by the components of dietary intake, and the current dietary recommendations are particularly beneficial for those who are genetically susceptible for obesity.

Key words: FTO gene: Diet: Obesity: Polymorphisms: Genetics

A common variant, rs9939609, of the fat mass and obesityassociated (FTO) gene has consistently shown a strong association with obesity $^{(1-3)}$. We have earlier confirmed the association between the FTO variant and BMI in the Finnish Diabetes Prevention Study (DPS $)^{(4)}$. Furthermore, we have reported that there was no association between rs9939609 and the magnitude of weight reduction achieved by a long-term lifestyle intervention in the DPS ${ }^{(4)}$. However, the extent to which lifestyle factors may modify this genetic risk is still quite unclear. Some studies have suggested that the 'obesogenic' effects of FTO may be accentuated by a highfat and low-carbohydrate diet ${ }^{(5)}$ or lower physical activity ${ }^{(6)}$, or blunted by higher physical activity ${ }^{(7)}$. These findings are suggestive for a role of lifestyle in modifying the association between FTO and obesity.

The discovery of FTO as an obesity-susceptibility gene has launched a series of studies to unravel the physiological mechanisms through which the risk alleles confer obesity. Major effects of FTO appear to be focused on energy intake instead of energy expenditure ${ }^{(8-11)}$. Children and adults carrying the

Abbreviations: DPS, Diabetes Prevention Study; E\%, percentage of energy.

*Corresponding author: Dr T. Lappalainen, fax +358 17163 2792, email tiina.lappalainen@uef.fi 
FTO risk allele have been reported to have higher energy intake ${ }^{(8-10,12,13)}$. Intake of all macronutrients has been shown to be greater in carriers of the risk allele but especially so for fat intake ${ }^{(9)}$. The abundant hypothalamic expression of FTO also supports a potential role in the control of satiety or appetite $^{(14-16)}$.

Long-term studies using high-quality, well-documented dietary data are warranted to further define how and to what degree the genetic risk by FTO is modified by dietary factors. Understanding the interactions between genetic variations and dietary intake would provide a basis for determining the role of dietary habits in the prevalence and pathogenesis of obesity.

\section{Methods}

\section{The Finnish Diabetes Prevention Study}

The Finnish DPS was a multicentre study carried out in 1993-2000 in five participating centres in Finland, located in Helsinki, Kuopio, Oulu, Tampere and Turku ${ }^{(17-19)}$. The main aim of the DPS was to assess the efficacy of an intensive diet and exercise programme to prevent or delay the onset of type 2 diabetes among high-risk individuals with impaired glucose tolerance. The main inclusion criteria were BMI over $25 \mathrm{~kg} / \mathrm{m}^{2}$ and age from 40 to 64 years. Impaired glucose tolerance was defined according to the WHO criteria $(1985)^{(20)}$. A total of 522 subjects were randomly assigned to an intensive diet and exercise counselling ( $n$ 265) or to a control ( $n$ 257) group. The main goals of the intervention group were weight reduction $\geq 5 \%$, moderate intensity physical activity $\geq 30 \mathrm{~min} / \mathrm{d}$, dietary fat $<30 \%$ of total energy ( $\mathrm{E} \%$ ), saturated fat $<10 \mathrm{E} \%$ and fibre $\geq 15 \mathrm{~g} / 4184 \mathrm{~kJ}$ (1000 kcal). Baseline characteristics by randomisation group are presented in Table 1. After a mean follow-up of 3.2 years, the risk of diabetes had reduced by $58 \%$ in the intervention group compared with the control group, and the intervention phase was terminated after a mean duration of 3.9 (range $0-6)$ years $^{(18,21)}$. In the present study, however, the intervention and control groups were analysed together and analyses were adjusted for the intervention. Biochemical and anthropometric measurements were performed at baseline and at the annual visits. The study protocol was approved by the
Ethics Committee of the National Public Health Institute in Helsinki. All participants volunteered for the study and gave their written informed consent.

\section{Assessment of dietary intake}

The study participants completed a $3 \mathrm{~d}$ food record at baseline and before each annual study visit ${ }^{(19,22)}$. They were asked to write down everything they ate and drank using a picture booklet of portion sizes of typical foods as the reference. The completeness of the food records was checked at a session with the study nutritionist during the study visit. The average annual nutrient intakes were calculated with a dietary analysis program developed at the National Public Health Institute ${ }^{(23)}$. In the present study, the average intakes at baseline and during the years 1,2 and 3 were used in the statistical analyses.

\section{Genotyping}

The original number of the study subjects in the DPS is 522 but all of them did not give permission for the genetic analyses, and thus the FTO variant rs9939609 was genotyped only from the available 479 DNA samples (160 men and 319 women). The marker did not deviate from Hardy-Weinberg equilibrium expectation $(P=0 \cdot 71)$. The custom Golden Gate genotyping reagents and consumables were ordered from Illumina, Inc. A representative sample (thirty-two replicates) was repeated to confirm the genotype and the success rate was $100 \%$

\section{Statistical analyses}

Statistical analyses were performed using SPSS statistical software for Windows (version 14.0; SPSS, Inc.). Results are presented as means and standard deviations. Normality of variable distributions were tested using the KolmogorovSmirnov test with Lilliefors correction. Logarithmic transformations were used to transform variables to normal distribution when appropriate. Homogeneity of variances was tested using Levene's test. Genetic associations with continuous variables were analysed with general linear model univariate ANOVA

Table 1. Baseline characteristics of the intervention and control groups in the Finnish Diabetes Prevention Study (original $n$ 522, in the present study $n$ 479)

(Mean values and standard deviations)

\begin{tabular}{|c|c|c|c|c|c|}
\hline & \multicolumn{2}{|c|}{$\begin{array}{l}\text { Intervention } \\
\text { group }\end{array}$} & \multicolumn{2}{|c|}{ Control group } & \multirow[b]{2}{*}{$P^{*}$} \\
\hline & Mean & SD & Mean & SD & \\
\hline$n$ & \multicolumn{2}{|c|}{241} & \multicolumn{2}{|c|}{238} & \\
\hline Sex (male/female) & \multicolumn{2}{|c|}{$84 / 157$} & \multicolumn{2}{|c|}{$76 / 162$} & 0.498 \\
\hline Age (years) & 55.4 & $7 \cdot 2$ & 54.9 & 6.9 & 0.401 \\
\hline Weight (kg) & $87 \cdot 0$ & $14 \cdot 2$ & $85 \cdot 5$ & $14 \cdot 2$ & 0.210 \\
\hline $\mathrm{BMI}\left(\mathrm{kg} / \mathrm{m}^{2}\right)$ & $31 \cdot 4$ & 4.6 & $31 \cdot 0$ & 4.4 & 0.420 \\
\hline Fasting plasma glucose $(\mathrm{mmol} / \mathrm{l})$ & $6 \cdot 1$ & 0.8 & $6 \cdot 2$ & 0.7 & 0.436 \\
\hline
\end{tabular}


at baseline and with linear mixed models during the 3 years of follow-up. Selected co-variables (sex, age, group and total energy intake) were included in the models as covariates. Normality was assessed by plotting the residuals. In the mixed models, subject identifiers were included as a grouping random effect. In the follow-up data analysis, the intervention and control groups were analysed together. After the interactions between the FTO genotype and a dietary factor were modelled, more specific analyses were performed according to the tertiles of dietary intake. Furthermore, a follow-up analysis was performed for the whole study population using BMI as the dependent variable and including all the dietary factors, sex, age, group and FTO SNP rs9939609 in the same mixed model.

To assess the statistical power of the study, power calculations were performed. Given the number of samples ( $n$ 479), minor allele frequency of $42.4 \%$, additive inheritance model, $\alpha$ significance level of 0.05 , estimated genetic effect of $0.1 \mathrm{BMI}$ units, and estimated environmental effect of 0.05 BMI units, we had $>80 \%$ power to detect gene-environment effects greater than $0.8 \mathrm{BMI}$ per one unit change in the interaction strength. Power calculations were performed using QUANTO 1.2.4 (http://hydra.usc.edu/gxe/).

\section{Results}

\section{Baseline analyses}

Associations between the FTO genotype and nutrient intakes at baseline are summarised in Table 2. FTO SNP rs9939609 was not associated with the mean values of total energy intake, or intakes of macronutrients or fibre. An additional adjustment for BMI did not change the results (data not shown). At baseline, those with the AA genotype had higher BMI than subjects with other genotypes (Table 2).

At baseline, higher BMI by the FTO risk genotype was detected especially in those who reported a diet high in saturated fat, with mean BMI of 30.4 (SD 3.6), 31.9 (SD 4.2) and $33.6(\mathrm{SD} 5.3) \mathrm{kg} / \mathrm{m}^{2}$ for TT, TA and AA carriers, respectively ( $P=0.004$, unadjusted; Table 3 ). The observed increase in BMI across the FTO genotypes was also restricted to those who reported a diet high in total fat (Fig. 1). Furthermore, higher BMI was observed in those who had a diet low in carbohydrates $(P=0.028)$ and fibre $(P=0.015$; Table 3$)$. However, in the analyses adjusted for total energy intake, age and sex, significant interactions between FTO and dietary intakes were not found. A trend for interaction $(P=0.097)$ was detected only for carbohydrates (Table 3). Total energy intake seemed to modify the association between the FTO genotype and BMI to a lower degree (Table 3).

\section{Follow-up analyses}

When the dietary intakes divided into tertiles were examined in relation to the FTO genotype and BMI, we observed that during the 3-year follow-up, individuals with the AA genotype did not differ from the other genotypes when they followed a diet low in energy (Fig. 2). However, the test for interaction between FTO and total energy intake was not significant $(P=0.086)$ after adjustment for sex, age and group. The additional adjustments for physical activity, smoking or recruitment centre did not change the results considerably (data not shown). The interactions between FTO and other dietary factors did not reach statistical significance either, but it appeared that the diets low in fat, high in carbohydrates or high in fibre were advantageous in relation to BMI for the risk allele carriers (Fig. 2).

In the linear mixed model including all the dietary factors, sex, age, group and FTO SNP rs9939609 predicting the BMI during the 3-year follow-up, FTO SNP $(P<0 \cdot 001)$, sex $(P<0.001)$, age $(P<0.001)$, SFA intake $(P<0.001)$ and protein intake $(P=0.020)$ remained significant predictors while adjusting for all the other variables.

The stratified analyses according to randomisation group were also performed, but there were no significant interactions between FTO and dietary factor (data not shown).

Table 2. Sex, BMI and nutrient intakes in the Finnish Diabetes Prevention Study according to the genotypes of rs9939609 of the fat mass and obesity-associated (FTO) gene

(Mean values and standard deviations)

\begin{tabular}{|c|c|c|c|c|c|c|c|c|}
\hline \multirow[t]{2}{*}{ rs9939609... } & \multicolumn{2}{|c|}{ TT } & \multicolumn{2}{|c|}{ TA } & \multicolumn{2}{|c|}{ AA } & \multirow{2}{*}{$\begin{array}{c}P^{*} \text { additive } \\
\text { model }\end{array}$} & \multirow{2}{*}{$\begin{array}{l}P \dagger \text { additive } \\
\text { model }\end{array}$} \\
\hline & Mean & SD & Mean & SD & Mean & SD & & \\
\hline Sex (male/female) & \multicolumn{2}{|c|}{$55 / 106$} & \multicolumn{2}{|c|}{$80 / 150$} & \multicolumn{2}{|c|}{$25 / 63$} & $0.542 \ddagger$ & \\
\hline BMI $\left(\mathrm{kg} / \mathrm{m}^{2}\right)$ & $30 \cdot 7$ & 4.4 & $31 \cdot 1$ & 4.4 & 32.6 & 4.7 & 0.004 & \\
\hline Total energy $(\mathrm{kJ} / \mathrm{d})$ & 7368 & 2079 & 7489 & 2268 & 7180 & 2004 & 0.726 & \\
\hline Fat $(E \%)$ & $36 \cdot 7$ & 6.9 & $36 \cdot 6$ & $6 \cdot 3$ & $37 \cdot 0$ & $6 \cdot 6$ & 0.985 & 0.650 \\
\hline SFA (E \%) & $16 \cdot 8$ & 4.0 & $16 \cdot 4$ & 4.0 & $17 \cdot 3$ & 4.4 & 0.261 & 0.168 \\
\hline MUFA (E\%) & $13 \cdot 2$ & 3.0 & $12 \cdot 9$ & $2 \cdot 6$ & $12 \cdot 9$ & $2 \cdot 9$ & 0.802 & 0.699 \\
\hline PUFA (E \%) & 5.9 & $2 \cdot 0$ & 5.8 & 1.9 & 5.5 & 1.8 & 0.496 & 0.533 \\
\hline Carbohydrates (E \%) & $43 \cdot 3$ & $7 \cdot 3$ & $43 \cdot 4$ & $6 \cdot 8$ & $42 \cdot 8$ & $6 \cdot 8$ & 0.611 & 0.510 \\
\hline Fibre $(\mathrm{g})$ & $19 \cdot 5$ & $7 \cdot 2$ & $20 \cdot 6$ & $7 \cdot 7$ & $18 \cdot 6$ & $6 \cdot 3$ & 0.109 & 0.147 \\
\hline
\end{tabular}

E\%, percentage of energy.

${ }^{*}$ ANOVA adjusted for age and sex.

†ANOVA adjusted for age, sex and total energy intake.

$\ddagger \chi^{2}$ test. 
Table 3. BMI by tertiles of dietary intake and fat mass and obesity-associated (FTO) genotype rs9939609 in the Finnish Diabetes Prevention Study at baseline

(Mean values and standard deviations)

\begin{tabular}{|c|c|c|c|c|c|c|c|c|c|c|c|c|c|c|}
\hline & \multirow[b]{2}{*}{ Mean intake } & \multirow[b]{2}{*}{ Range } & \multicolumn{3}{|c|}{$\mathrm{TT}$} & \multicolumn{3}{|c|}{ TA } & \multicolumn{3}{|c|}{$A A$} & \multirow[b]{2}{*}{$P^{*}$} & \multirow[b]{2}{*}{$P \dagger$} & \multirow[b]{2}{*}{$P \ddagger$} \\
\hline & & & Mean & SD & $n$ & Mean & SD & $n$ & Mean & SD & $n$ & & & \\
\hline \multicolumn{15}{|l|}{ Energy (kJ) } \\
\hline Low & 5268 & $2377-6263$ & $30 \cdot 7$ & $3 \cdot 8$ & 59 & $31 \cdot 7$ & $3 \cdot 8$ & 70 & 32.5 & 5.9 & 30 & 0.197 & 0.857 & 0.856 \\
\hline Medium & 7096 & $6268-7987$ & $30 \cdot 8$ & 4.4 & 45 & $30 \cdot 8$ & 4.3 & 80 & $32 \cdot 7$ & $3 \cdot 7$ & $35 \S$ & 0.035 & 0.402 & \\
\hline High & 9816 & $7987-16385$ & 30.3 & $4 \cdot 7$ & 56 & 30.8 & 5.0 & 80 & $32 \cdot 6$ & 4.6 & 23 & 0.124 & 0.832 & \\
\hline \multicolumn{15}{|l|}{ Fat (E\%) } \\
\hline Low & $29 \cdot 6$ & $19 \cdot 1-34 \cdot 2$ & $30 \cdot 3$ & 3.3 & 56 & 30.4 & $4 \cdot 1$ & 76 & $31 \cdot 6$ & 3.4 & 27 & 0.242 & 0.786 & 0.164 \\
\hline Medium & $36 \cdot 7$ & $34.2-39.8$ & 30.9 & 5.4 & 48 & 31.5 & 4.4 & 76 & $32 \cdot 0$ & $4 \cdot 1$ & 36 & 0.399 & 0.480 & \\
\hline High & $43 \cdot 8$ & $39 \cdot 8-61 \cdot 1$ & $30 \cdot 6$ & $4 \cdot 1$ & 56 & $31 \cdot 3$ & $4 \cdot 6$ & 78 & 34.5 & $6 \cdot 2$ & $25 \S$ & 0.005 & 0.099 & \\
\hline \multicolumn{15}{|l|}{ SFA (E\%) } \\
\hline Low & $12 \cdot 3$ & $7 \cdot 1-14 \cdot 8$ & $30 \cdot 2$ & $4 \cdot 0$ & 58 & $30 \cdot 8$ & $4 \cdot 7$ & 82 & $31 \cdot 1$ & $3 \cdot 1$ & 19 & 0.630 & 0.855 & 0.145 \\
\hline Medium & $16 \cdot 7$ & $14 \cdot 8-18 \cdot 5$ & 31.3 & $5 \cdot 1$ & 50 & $30 \cdot 6$ & 4.2 & 75 & 32.5 & $4 \cdot 8$ & 35 & 0.120 & 0.627 & \\
\hline High & $21 \cdot 2$ & $18 \cdot 5-29 \cdot 7$ & 30.4 & 3.6 & 52 & 31.9 & $4 \cdot 2$ & $73 \S$ & 33.6 & $5 \cdot 3$ & $34 \S$ & 0.004 & 0.447 & \\
\hline \multicolumn{15}{|l|}{$\mathrm{CHO}(\mathrm{E} \%)$} \\
\hline Low & $35 \cdot 7$ & $16 \cdot 8-40 \cdot 3$ & $31 \cdot 2$ & $4 \cdot 3$ & 55 & 31.9 & 4.6 & 73 & $34 \cdot 1$ & $6 \cdot 1$ & $31 \S$ & 0.028 & 0.051 & 0.097 \\
\hline Medium & $43 \cdot 2$ & $40 \cdot 4-46 \cdot 4$ & $30 \cdot 1$ & $4 \cdot 2$ & 50 & 31.0 & 4.5 & 82 & $32 \cdot 0$ & 3.9 & 28 & 0.107 & 0.442 & \\
\hline High & $51 \cdot 0$ & $46 \cdot 4-68 \cdot 6$ & 30.5 & 4.4 & 55 & 30.3 & $4 \cdot 1$ & 75 & 31.5 & $3 \cdot 4$ & 29 & 0.331 & 0.527 & \\
\hline \multicolumn{15}{|l|}{ Fibre (g) } \\
\hline Low & $12 \cdot 7$ & $4 \cdot 4-16 \cdot 2$ & 31.3 & $4 \cdot 1$ & 57 & 31.4 & 3.9 & 69 & $34 \cdot 1$ & 5.9 & $33 \S$ & 0.015 & 0.641 & 0.482 \\
\hline Medium & $19 \cdot 0$ & $16 \cdot 2-22 \cdot 2$ & $29 \cdot 9$ & $4 \cdot 0$ & 53 & $30 \cdot 6$ & $3 \cdot 8$ & 74 & 31.9 & 4.0 & 33 & 0.070 & 0.362 & \\
\hline High & $27 \cdot 9$ & $22 \cdot 3-64 \cdot 1$ & 30.5 & $4 \cdot 8$ & 50 & $31 \cdot 1$ & $5 \cdot 2$ & 87 & 31.5 & 3.0 & 22 & 0.571 & 0.734 & \\
\hline \multicolumn{15}{|c|}{ Protein (E\%) } \\
\hline Low & 14.4 & $9 \cdot 0-15 \cdot 7$ & 30.4 & 4.3 & 58 & 31.2 & $4 \cdot 6$ & 70 & 33.0 & 4.9 & $31 \S$ & 0.039 & 0.461 & 0.965 \\
\hline Medium & $16 \cdot 9$ & $15 \cdot 7-18 \cdot 4$ & $30 \cdot 1$ & $4 \cdot 2$ & 47 & 30.2 & 3.9 & 81 & $32 \cdot 4$ & 4.7 & $32 \S$ & 0.020 & 0.011 & \\
\hline High & 21.4 & $18 \cdot 4-33.3$ & $31 \cdot 3$ & $4 \cdot 2$ & 55 & 31.8 & 4.6 & 79 & $32 \cdot 3$ & $4 \cdot 7$ & 25 & 0.586 & 0.220 & \\
\hline
\end{tabular}

$\mathrm{E} \%$, percentage of energy; $\mathrm{CHO}$, carbohydrates.

${ }^{*}$ Differences in BMI of an additive genetic model by general linear model.

†Differences in BMI of an additive genetic model by general linear model adjusted for sex, age and total energy intake.

$\ddagger$ Interaction: genotype $\times$ dietary factor (as a continuous variable) when adjusted for sex, age and total energy intake.

$\S$ TT v. TA/AA, $P<0.05$.

\section{Discussion}

Understanding the interactions between dietary intake and genetic variation provides a basis for determining the role of dietary habits in the prevalence and pathogenesis of obesity. In the present study, we suggested that at baseline and during the follow-up, the association between FTO and BMI was more pronounced in those having a diet high in fat and low in carbohydrates and fibre. Although the FTO $\times$ diet interactions did not reach conventional statistical significance $(P<0.05)$ in the present study, we would like to highlight the importance of detected trends. One could argue the relevance of the present findings because of the absence of interaction, i.e. it appears that also non-risk allele carriers of FTO rs9939609 benefited in relation to BMI from a diet low in energy and fat, but high in carbohydrate and fibre (Fig. 2). However, the present findings emphasise the importance of the same diet for the risk allele carriers of FTO, as well. In the whole study population, the mean values for the lowest tertiles of fat intake were $29 \cdot 6,26 \cdot 5$ and $26 \cdot 1 \mathrm{E} \%$, and for saturated fat $12.3,10.2$ and $9.8 \mathrm{E} \%$ at baseline, at the first and third year, respectively. Corresponding values for the highest tertiles of daily fibre intake were $28,28.5$ and $28.5 \mathrm{~g}$. The mean values of the lowest tertiles of reported carbohydrate intake were $35.7,37.6$ and $38.3 \mathrm{E} \%$. Thus, we consider that the present results highlight the validity of the current dietary recommendations especially for those people with known genetic risk of obesity: reducing the intake of total and saturated fat and increasing the relative intake of carbohydrates rich in fibre. Typically, reduction in energy intake can also be achieved by following these guidelines ${ }^{(24)}$. Previously, Lindström et $a l^{(22)}$ have likewise shown that a long-term weight loss and concomitant type 2 diabetes risk reduction was best achieved by increasing fibre intake and decreasing the intake of dietary fat in the Finnish DPS.

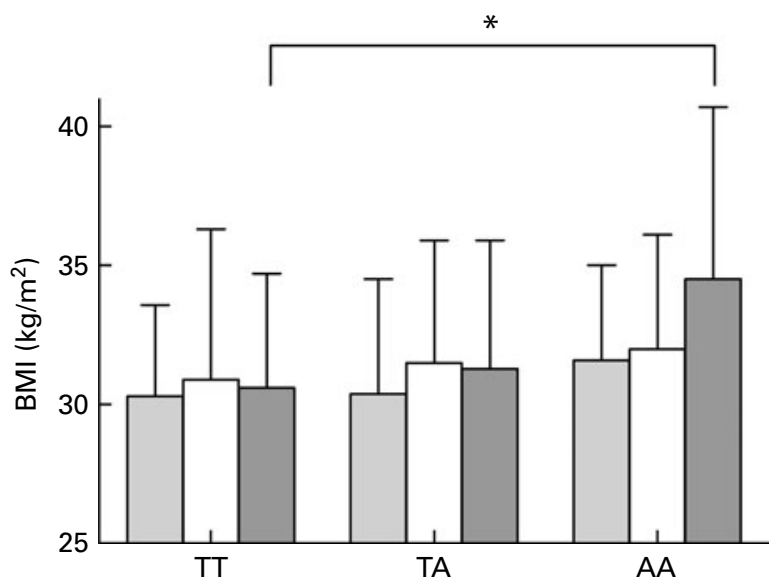

Fig. 1. BMI by dietary fat intake tertiles (low ( $\square$ ), medium ( $\square$ ) and high $(\square)$ ) and by fat mass and obesity-associated (FTO) genotype rs9939609 at baseline. ${ }^{\star} P<0.05$. 
(a)
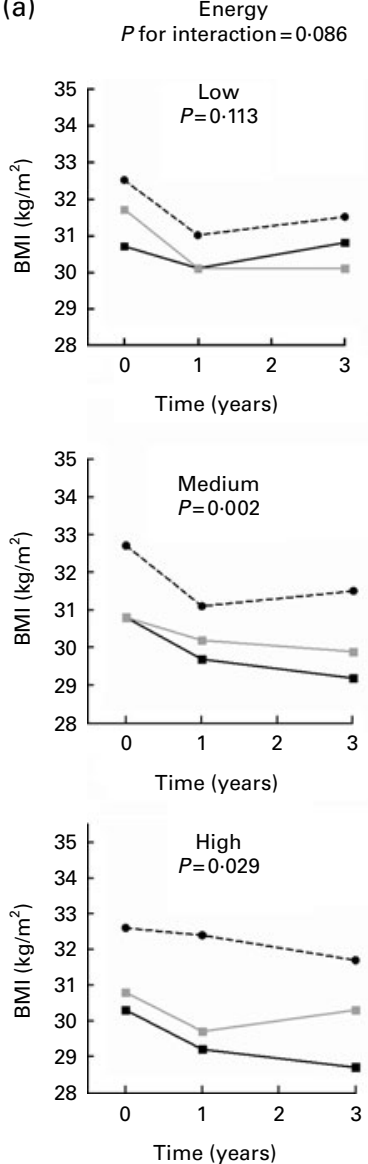

(b)
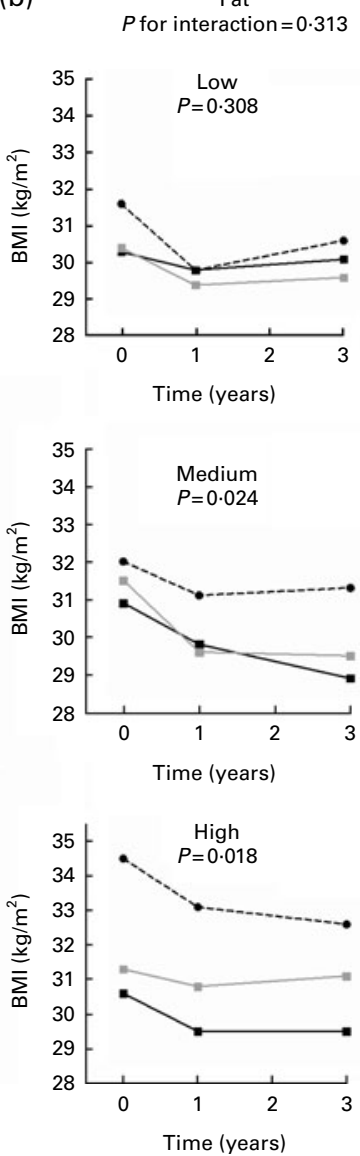

(c)
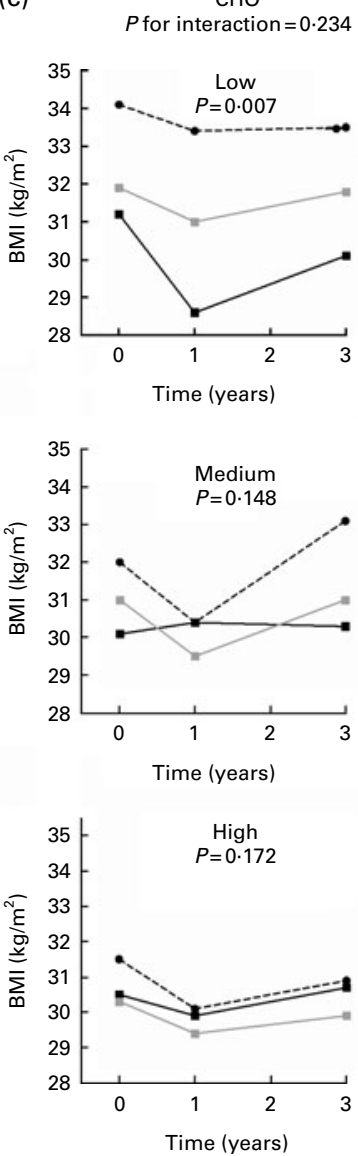

(d)
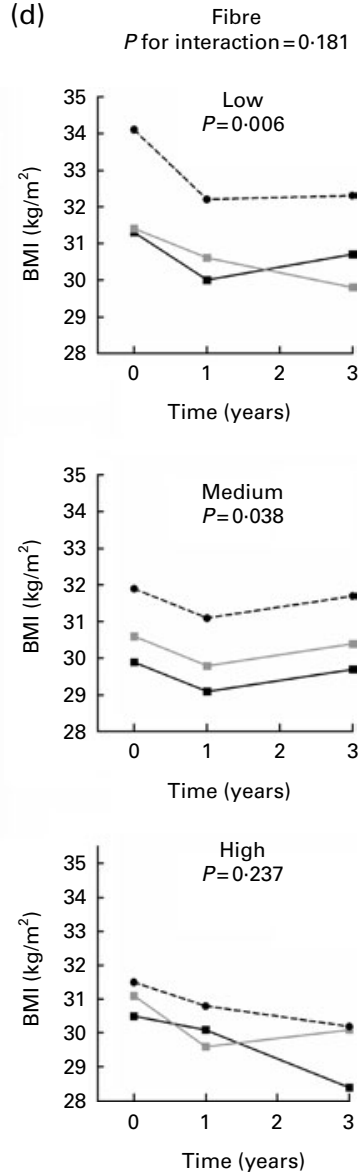

Fig. 2. BMI by (a) energy, (b) fat, (c) carbohydrate $(\mathrm{CHO})$ and (d) fibre intake tertiles (low, medium and high) and by fat mass and obesity-associated (FTO) genotype rs9939609 during the 3-year follow-up. Analyses are adjusted for total energy intake (for (b) $-(\mathrm{d})$ ), sex, age and group. The $P$ value for interaction between the FTO genotype and a dietary factor on BMI was first modelled. Then data were divided into tertiles according to dietary intake and comparisons between the genotype groups were analysed within the tertile groups. $\_-, \mathrm{TT} ; \ldots$

The present findings are in accordance with two recent reports ${ }^{(5,25)}$. A study by Sonestedt et al. ${ }^{(5)}$ was based on an analysis of cross-sectional data, and they showed similarly that an increase in BMI across FTO genotypes was restricted to those who reported a high-fat diet. In addition, they observed higher BMI for AA carriers of FTO rs9939609 among individuals with a low carbohydrate intake. However, they did not observe an interaction between fibre intake and the FTO genotype on BMI. Moleres et al. ${ }^{(25)}$ have shown in children that risk allele carriers consuming saturated fat more than $12.6 \mathrm{E} \%$ had an increased obesity risk compared with other genotype carriers. Furthermore, Razquin et al. $^{(26)}$ have demonstrated that a Mediterranean-based diet, rich in mono- and polyunsaturated fat, provides FTO risk allele carriers some protection against bodyweight gain after 3 years of nutritional intervention. Very recently, Ahmad et al. ${ }^{(27)}$ noted larger effects of the FTO risk allele on BMI in women who were less active or had higher energy intake. These and the present results support the hypothesis that the BMI-increasing effect of FTO is higher in a population with a Western lifestyle characterised by an energy-dense diet and a sedentary lifestyle, but the mechanisms behind this still remain to be explained. In general, these observations highlight the multi-factorial nature of obesity, each individual genetic variant has probably only a modest effect, and the interaction of genetic variants with environmental factors can potentially be quite important in determining the observed phenotype.

In the previous studies, the abundant FTO expression in the brain areas governing energy balance has supported a central neuronal role for $F T O^{(14,16,28)}$. Studies in human subjects have underpinned this hypothesis, since the FTO locus has been shown to confer a risk of obesity through increased energy intake and reduced satiety, although several negative findings have been reported in this regard as well. Previous studies on the association between FTO polymorphism and dietary intake and eating behaviour are summarised in Table 4. In the present study, FTO SNP rs9939609 was not associated with the mean values of total energy intake, macronutrients or fibre at baseline. Most of the previous studies reporting an effect of FTO on elevated dietary intake were conducted in children ${ }^{(8,9,12)}$, which may explain the discrepancy. However, by which exact mechanism FTO could particularly influence children's eating behaviour still needs further investigation.

In the present study, the obvious limitation is a small sample size in terms of interaction analyses, although power 
Table 4. Studies on the association between fat mass and obesity-associated (FTO) polymorphism and dietary intake and eating behaviour

\begin{tabular}{|c|c|c|c|c|}
\hline Reference & FTO SNP & Subjects & Study design & Effect \\
\hline Ahmad et al. ${ }^{(27)}$ & rs8050136 & 21675 adults & Observational follow-up & $\begin{array}{l}\text { Risk allele carriers had larger effects on BMI if } \\
\text { they were less active or had higher energy intake }\end{array}$ \\
\hline Cecil et al. ${ }^{(9)}$ & rs9939609 & 97 children & $\begin{array}{l}\text { Cross-sectional } \\
\text { population-based }\end{array}$ & Association with increased energy intake \\
\hline den Hoed et al. ${ }^{(29)}$ & rs9939609 & 103 adults & Intervention study & $\begin{array}{l}\text { Association with low postprandial responses in } \\
\text { hunger and satiety }\end{array}$ \\
\hline Do et al. ${ }^{(30)}$ & $\begin{array}{l}\text { rs } 17817449 \\
\text { rs1421085 }\end{array}$ & 908 adults & $\begin{array}{l}\text { Cross-sectional population-based, } \\
\text { family study }\end{array}$ & No BMI-independent association in energy intake \\
\hline Hakanen et al. ${ }^{(31)}$ & rs9939609 & 438 children & $\begin{array}{l}\text { Prospective, population-based } \\
\text { intervention }\end{array}$ & No association with energy intake \\
\hline Haupt et al. ${ }^{(10)}$ & rs 8050136 & 380 adults & Lifestyle intervention programme & $\begin{array}{l}\text { Association with higher energy intake during } \\
\text { dietary restriction among risk allele carriers }\end{array}$ \\
\hline Razquin et al. ${ }^{(26)}$ & rs9939609 & 776 adults & Lifestyle intervention programme & $\begin{array}{l}\text { Mediterranean-style diet protected risk allele } \\
\text { carriers from weight gain }\end{array}$ \\
\hline Sonestedt et al. ${ }^{(5)}$ & rs9939609 & 4839 adults & Cross-sectional population-based & $\begin{array}{l}\text { The increase in BMI across genotypes was } \\
\text { restricted to those who reported a high-fat diet }\end{array}$ \\
\hline Speakman et al..$^{(32)}$ & rs9939609 & 150 adults & Cross-sectional population-based & Association with variation in energy intake \\
\hline Tanofsky-Kraff et al. ${ }^{(33)}$ & rs9939609 & 289 children & Cross-sectional population-based & $\begin{array}{l}\text { Subjects with risk alleles were more likely to report } \\
\text { episodes of loss of control of eating and they } \\
\text { consumed a greater percentage of energy from fat }\end{array}$ \\
\hline Timpson et al. ${ }^{(12)}$ & rs9939609 & 3641 children & Cross-sectional population-based & $\begin{array}{l}\text { Subjects with risk alleles consumed more fat } \\
\text { and total energy }\end{array}$ \\
\hline Wardle et al. ${ }^{(13)}$ & rs9939609 & 3337 children & Cross-sectional population-based & $\begin{array}{l}\text { Subjects with risk alleles had lower satiety } \\
\text { responsiveness }\end{array}$ \\
\hline Wardle et al. ${ }^{(8)}$ & rs9939609 & 131 children & Cross-sectional population-based & $\begin{array}{l}\text { Subjects with risk alleles showed higher } \\
\text { consumption of a highly palatable food }\end{array}$ \\
\hline
\end{tabular}

calculations indicated the statistical power to be relatively good and this is explained most probably by the high minor allele frequency of FTO rs9939609. However, we acknowledge the need for larger studies to determine the exact nature of the interaction. Furthermore, we would like to emphasise that the strength of the DPS population is that it is carefully selected and clinically well characterised. Furthermore, the habitual nutrient intakes of the study participants were annually estimated using $3 \mathrm{~d}$ food records, which is a reliable and high-quality method in analysing dietary intakes. It is also worth noting that the present study enables the investigation of long-term gene $x$ environment interactions, and this kind of data is seldom available.

The variables used to control for in the statistical analyses were age, sex group, total energy intake and randomisation group. The additional adjustments for physical activity, smoking or recruitment centre did not change the results considerably (data not shown). Furthermore, the stratified analyses according to randomisation group were performed, but there were no significant interactions between FTO and dietary factors (data not shown).

Taken together, the present study strengthens the view that diet plays a role in the association between the genetic variants of FTO and obesity. Thus, current dietary recommendations are particularly beneficial for those who are genetically susceptible for obesity.

\section{Acknowledgements}

This study was supported by grants from the Sigrid Juselius Foundation, the Academy of Finland (no. 118590), the EVO funding of the Kuopio University Hospital from Ministry of
Health and Social Affairs (no. 5254), the Finnish Funding Agency for Technology and Innovation (40058/07) and the Nordic Centre of Excellence on 'Systems biology in controlled dietary interventions and cohort studies', SYSDIET (no. 070014). T. L., J. L., J. T., J. P., L. K., J. G. E. and M. U. designed the study; M. U. and J. T. conducted the research; T. L. analysed the data, performed the statistical analyses and wrote the manuscript; T. L. had primary responsibility for the final content. All authors critically reviewed the manuscript, and read and approved the final manuscript. None of the authors reported a conflict of interest.

\section{References}

1. Frayling TM, Timpson NJ, Weedon MN, et al. (2007) A common variant in the FTO gene is associated with body mass index and predisposes to childhood and adult obesity. Science 316, 889-894.

2. Scuteri A, Sanna S, Chen WM, et al. (2007) Genome-wide association scan shows genetic variants in the FTO gene are associated with obesity-related traits. PLoS Genet 3, e115.

3. Dina C, Meyre D, Gallina S, et al. (2007) Variation in FTO contributes to childhood obesity and severe adult obesity. Nat Gene 39, 724-726.

4. Lappalainen TJ, Tolppanen AM, Kolehmainen M, et al. (2009) The common variant in the FTO gene did not modify the effect of lifestyle changes on body weight: the Finnish Diabetes Prevention Study. Obesity (Silver Spring) 17, 832-836.

5. Sonestedt E, Roos C, Gullberg B, et al. (2009) Fat and carbohydrate intake modify the association between genetic variation in the FTO genotype and obesity. Am J Clin Nutr 90, 1418-1425.

6. Andreasen $\mathrm{CH}$, Stender-Petersen $\mathrm{KL}$, Mogensen MS, et al. (2008) Low physical activity accentuates the effect of the 
FTO rs9939609 polymorphism on body fat accumulation. Diabetes 57, 95-101.

7. Rampersaud E, Mitchell BD, Pollin TI, et al. (2008) Physical activity and the association of common FTO gene variants with body mass index and obesity. Arch Intern Med 168, $1791-1797$.

8. Wardle J, Llewellyn C, Sanderson S, et al. (2009) The FTO gene and measured food intake in children. Int $J$ Obes (Lond) 33, 42-45.

9. Cecil JE, Tavendale R, Watt P, et al. (2008) An obesityassociated $F T O$ gene variant and increased energy intake in children. N Engl J Med 359, 2558-2566.

10. Haupt A, Thamer C, Staiger H, et al. (2009) Variation in the FTO gene influences food intake but not energy expenditure. Exp Clin Endocrinol Diabetes 117, 194-197.

11. Berentzen T, Kring SI, Holst C, et al. (2008) Lack of association of fatness-related FTO gene variants with energy expenditure or physical activity. J Clin Endocrinol Metab 93, 2904-2908.

12. Timpson NJ, Emmett PM, Frayling TM, et al. (2008) The fat mass- and obesity-associated locus and dietary intake in children. Am J Clin Nutr 88, 971-978.

13. Wardle J, Carnell S, Haworth CM, et al. (2008) Obesity associated genetic variation in FTO is associated with diminished satiety. J Clin Endocrinol Metab 93, 3640-3643.

14. Willer CJ, Speliotes EK, Loos RJ, et al. (2009) Six new loci associated with body mass index highlight a neuronal influence on body weight regulation. Nat Genet 41, 25-34.

15. Gerken T, Girard CA, Tung YC, et al. (2007) The obesityassociated FTO gene encodes a 2-oxoglutarate-dependent nucleic acid demethylase. Science 318, 1469-1472.

16. Stratigopoulos G, Padilla SL, LeDuc CA, et al. (2008) Regulation of Fto/Ftm gene expression in mice and humans. Am J Physiol Regul Integr Comp Physiol 294, R1185-R1196.

17. Eriksson J, Lindstrom J, Valle T, et al. (1999) Prevention of type II diabetes in subjects with impaired glucose tolerance: the Diabetes Prevention Study (DPS) in Finland. Study design and 1-year interim report on the feasibility of the lifestyle intervention programme. Diabetologia 42, 793-801.

18. Tuomilehto J, Lindstrm J, Eriksson JG, et al. (2001) Prevention of type 2 diabetes mellitus by changes in lifestyle among subjects with impaired glucose tolerance. $N$ Engl $J$ Med 344, 1343-1350.

19. Lindström J, Louheranta A, Mannelin M, et al. (2003) The Finnish Diabetes Prevention Study (DPS): lifestyle intervention and 3 -year results on diet and physical activity. Diabetes Care 26, 3230-3236.

20. WHO Study Group (1985) Diabetes mellitus. Report of $a$ WHO Study Group. World Health Organization Technical Report Series no. 727, pp. 113.
21. Lindström J, Ilanne-Parikka P, Peltonen M, et al. (2006) Sustained reduction in the incidence of type 2 diabetes by lifestyle intervention: follow-up of the Finnish Diabetes Prevention Study. Lancet 368, 1673-1679.

22. Lindström J, Peltonen M, Eriksson JG, et al. (2006) High-fibre, low-fat diet predicts long-term weight loss and decreased type 2 diabetes risk: the Finnish Diabetes Prevention Study. Diabetologia 49, 912-920.

23. Ovaskainen M-L, Valsta L \& Lauronen J (1996) The compilation of food analysis values as a database for dietary studies - the Finnish experience. Food Chem 57, 133-136.

24. Poppitt SD (1995) Energy density of diets and obesity. Int J Obes Relat Metab Disord 19, S20-S26.

25. Moleres A, Ochoa MC, Rendo-Urteaga T, et al. (2012) Dietary fatty acid distribution modifies obesity risk linked to the rs9939609 polymorphism of the fat mass and obesityassociated gene in a Spanish case-control study of children. Br J Nutr 107, 533-538.

26. Razquin C, Martinez JA, Martinez-Gonzalez MA, et al. (2010) A 3-year intervention with a Mediterranean diet modified the association between the rs9939609 gene variant in FTO and body weight changes. Int J Obes (Lond) 34, 266-272.

27. Ahmad T, Lee IM, Paré G, et al. (2011) Lifestyle interaction with fat mass and obesity-associated (FTO) genotype and risk of obesity in apparently healthy U.S. women. Diabetes Care 34, 675-680.

28. Fredriksson R, Hägglund M, Olszewski PK, et al. (2008) The obesity gene, FTO, is of ancient origin, up-regulated during food deprivation and expressed in neurons of feeding-related nuclei of the brain. Endocrinology 149, 2062-2071.

29. den Hoed M, Westerterp-Plantenga MS, Bouwman FG, et al. (2009) Postprandial responses in hunger and satiety are associated with the rs9939609 single nucleotide polymorphism in FTO. Am J Clin Nutr 90, 1426-1432.

30. Do R, Bailey SD, Desbiens K, et al. (2008) Genetic variants of FTO influence adiposity, insulin sensitivity, leptin levels, and resting metabolic rate in the Quebec Family Study. Diabetes 57, 1147-1150.

31. Hakanen M, Raitakari OT, Lehtimaki T, et al. (2009) FTO genotype is associated with body mass index after the age of seven years but not with energy intake or leisure-time physical activity. J Clin Endocrinol Metab 94, 1281-1287.

32. Speakman JR, Rance KA \& Johnstone AM (2008) Polymorphisms of the FTO gene are associated with variation in energy intake, but not energy expenditure. Obesity (Silver Spring) 16, 1961-1965.

33. Tanofsky-Kraff M, Han JC, Anandalingam K, et al. (2009) The FTO gene rs9939609 obesity-risk allele and loss of control over eating. Am J Clin Nutr 90, 1483-1488. 